\title{
Worst Expected Ramp Rates from Cloud Speed Measurements
}

\author{
Guang Chao Wang*, Juan Luis Bosch ${ }^{\dagger}$, Ben Kurtz*, Íñigo de la Parra ${ }^{\ddagger}$, Elynn Wu* and Jan Kleissl* \\ ${ }^{*}$ Center for Energy Research, University of California San Diego, La Jolla, California 92093, United States \\ ${ }^{\dagger}$ Department of Chemistry and Physics, University of Almera, 04120 Almera, Spain \\ $\ddagger$ Institute of Smart Cities, Public University of Navarre, Arrosadia Campus, 31006 Pamplona, Spain
}

\begin{abstract}
Large PV power ramp rates are of concern and sometimes even explicitly restricted by grid operators. Battery energy storage systems can smooth the power output and maintain ramp rates within permissible limits. To enable PV plant and energy storage systems design and planning, a method to estimate the largest expected ramps for a given location is proposed. Because clouds are the dominant source of PV power output variability, an analytical relationship between the worst expected ramp rates, cloud motion vectors, and the geometrical layout of the PV plant is developed. The ability of the proposed method to bracket actual ramp rates is assessed over 8 months under different meteorological conditions, demonstrating an average compliance rate of $96.9 \%$ for a 2 min evaluation time window.

Index Terms-Cloud Speed Sensor, Power Ramp Rate Estimate, PV Plant Design.
\end{abstract}

\section{INTRODUCTION}

The power output variability by large-scale grid-connected photovoltaic (PV) systems can negatively affect power quality and grid network reliability. Regulations have been introduced to restrict the maximum power ramp rates for PV plants on 1 min timescales [1]. These restrictions typically invite one of two approaches: (1) Compensate the power variability through energy storage systems (ESS). The storage requirements and strategies to comply with the regulations, considering capacity losses and cycling degradation, have been comprehensively studied [2], [3]. (2) Curtail the PV output to smooth up-ramps reactively and provide a buffer for smoothing down-ramps proactively [4], [5]. For example, short-term forecasts for future cloud arrivals allow a system operator to meet ramp rate restrictions with less battery reserve or curtailment [6], [7]. If all ramp rates (except plant outages) were to be mitigated, approach (1) would require knowledge of the worst-case ramp rate to determine the power and energy rating of the ESS. Given perfect forecasts, approach (2) could mitigate all power ramps without ESS. In practice, however, significant errors in the short-term ramp forecasts combined with restrictive ramp rate compliance requirements typically still require a ESS to mitigate worst-case ramp rates, but accurate forecasts can reduce the number of charge/discharge cycles of the ESS.

The nature of PV power variability has been well-studied [8]-[11]. For example, Marcos et al. [12] studied the smoothing effect of power fluctuations over the area of the power plant by low-pass filtering irradiance measurements at a single point. Lave et al. [13] proposed a wavelet variability model to simulate the reduction in power output fluctuations of a plant or a fleet of dispersed plants. The correlation scaling coefficient introduced in the model is universal and a function of cloud speed [14]. Marcos et al. [15] simulated the power output by a fleet of plants, using only irradiance measurements at a single location and the smoothing effect due to geographical dispersion and plant size. The solar power variability models in the aforementioned studies require high frequency solar irradiance measurements. While high frequency solar irradiance data are rarely available, some applications, such as PV plant design and ESS sizing, require only the worst power fluctuations. For example, the worst ramp rate determines the required power and energy capacity rating for the ESS to buffer all down-ramps. Motivated by this, we propose a novel analytical approach, where the maximum expected PV ramp rate is computed in a processbased model using: 1) low-resolution point irradiance or PV power measurements, 2) the geometrical layout of PV plant, and 3) cloud velocity. The main contribution of this paper is the derivation and demonstration of a simple but universally applicable model that bounds the maximum ramp rate using simple data input even in the absence of high temporal resolution.

The remainder of this paper is organized as follows. Sections II-A and III-B present the ramp rate model as an analytical relation between cloud velocity, PV plant dimension, recent PV power measurements / cloud optical depth, and expected maximum ramp rate. Section III-C introduces metrics to evaluate the model. Section III-A describes the experimental setup and data, and Section III-B introduces the process to derive the data input for the model. Sections IV-A and IV-B examine the model performance through detailed analysis for two example days and validation over the 8-month data collection period. Limitations of the proposed methodology and discussion are given in Section IV-C Lastly, Section V provides the conclusions.

\section{Mathematical Derivation And Problem FORMULATION}

\section{A. A Geometric Ramp Rate Model}

The model is based on the following five assumptions:

1) A steady irradiance field that is larger than the PV plant.

2) The clear and cloudy parts of the irradiance field are homogeneous and steady within the time interval of interest. 
3) No cloud edge effects such that clear and cloudy irradiance values are separated by a line.

4) No mismatch losses in the PV plant, i.e. PV plant power is proportional to spatially averaged irradiance.

5) Constant PV efficiency, i.e. no cell temperature or inverter effects.

With these assumptions, a power ramp results solely from the movement of the irradiance field over the PV plant, as conceptualized in Figure 1. The ramp rate is modeled based on the interaction between the irradiance field advected by the CMV and the PV plant geometry. At the initial time $t_{0}$, a portion of the irradiance field (bordered by the black rectangle that marks the PV plant size) affects the PV plant. The portion of the irradiance field that covers the plant at $t_{0}$ is then advected during a small time interval $\Delta t$ into a new position that is bordered by the grey dashed rectangle. During the advection, part of the irradiance field (the grey area, denoted as the outgoing portion of size $\Delta S$ ) moves off the plant while another part moves in (the red area, denoted as the incoming portion also of size $\Delta S$ ). $\Delta S$ can be computed as:

$$
\Delta S=(L v|\cos \alpha|+W v|\sin \alpha|) \Delta t-(v \Delta t)^{2}|\sin \alpha \cos \alpha|
$$

where $L$ and $W$ are the dimensions of the PV plant, and $v$ and $\alpha$ are the speed and direction of the irradiance field, respectively. The cloud field properties (i.e. speed, direction, and optical thickness) are reasonably assumed stationary within the time interval $\Delta t$. The difference in cloud optical thickness between the outgoing and incoming $\Delta S$ induces a power ramp event.

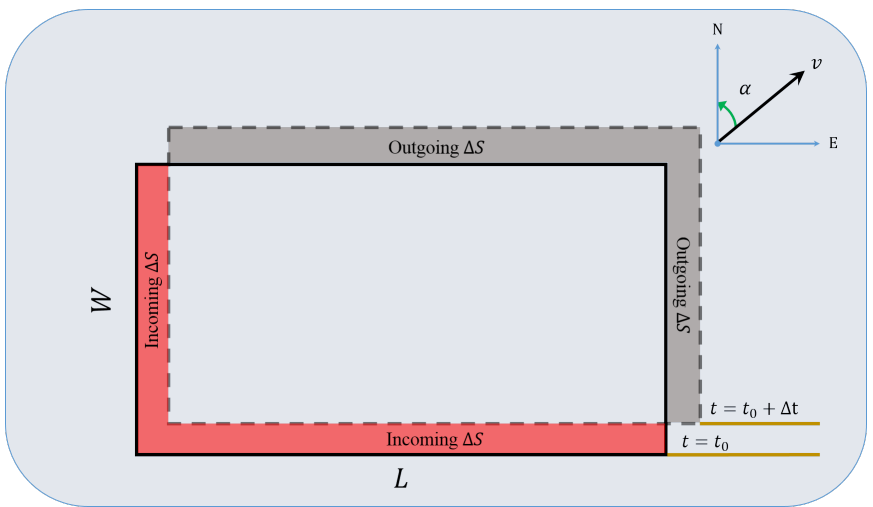

Fig. 1. The irradiance field (outer round rectangle containing clouds and clear sky) over the PV plant at $t_{0}$ is advected by speed $v$ and direction $\alpha$. The portion of the irradiance field that covered the plant (black rectangle) moves to a new location (grey rectangle) in time interval $\Delta t$, resulting in incoming and outgoing portions of the irradiance field with a size of $\Delta S$. The PV plant measures $L$ by $W$ and aligns with the East and North directions.

For reference, the area-normalized clear sky power production $\hat{P}_{c s}$ is calculated by 2 given the modeled or measured power production under clear sky condition $P_{c s}$ and PV plant dimensions $L$ and $W$ :

$$
\hat{P}_{c s}=\frac{P_{c s}}{L W}
$$

The clear sky PV power for a given area is then assumed to be proportional to $P_{c s}$. Thus, the solar power that would be produced by the net area $\Delta S$ under clear sky condition can be expressed as:

$$
P^{*}=\hat{P}_{c s} \Delta S \text {. }
$$

Not only a faster cloud field causes larger power reduction, but also a thicker cloud field would result in a larger ramp event. To factor in the solar power reduction caused by the cloud optical thickness, the clear sky index $k t[16]$ is included to reflect a power reduction scale under cloudy condition. The solar power change $\Delta P$ is:

$$
\Delta P=\left(k t_{i}-k t_{o}\right) P^{*},
$$

where $k t_{i}$ and $k t_{o}$ respectively represent the average cloud optical thickness for the incoming portion (red colored area in Figure 1) and the outgoing portion (complementary grey colored area) of the irradiance field. Since the irradiance field is a mix of cloudy and clear sky conditions, $k t_{i}-k t_{o}$ can be positive or negative. Finally, the ramp rate $R R$ becomes:

$$
\begin{aligned}
R R=\frac{\Delta P}{\Delta t}= & \pm(L v|\cos \alpha|+W v|\sin \alpha|- \\
& \left.v^{2} \Delta t|\sin \alpha \cos \alpha|\right) \frac{P_{c s}\left|k t_{i}-k t_{o}\right|}{L W} .
\end{aligned}
$$

\section{B. The Worst-Case Scenario Ramp Rate (WCS-RR)}

Equation (5) is not intended for operational ramp rate forecasts since without a sky imager the upwind irradiance field that would be needed to quantify $k t_{i}$ is generally not available. Instead, we consider the worst-case scenario, where a clear sky gives way to an overcast sky. We estimate the largest ramp rate by picking the smallest $k t$ from recent history (e.g. $30 \mathrm{~min}) k t_{\min }$. The WCS-RR can be expressed as:

$$
\begin{aligned}
\mathrm{WCS}-\mathrm{RR}= & \pm(L v|\cos \alpha|+W v|\sin \alpha|- \\
& \left.v^{2} \Delta t|\sin \alpha \cos \alpha|\right) \frac{P_{c s}\left|1-k t_{\text {min }}\right|}{L W} .
\end{aligned}
$$

In this formulation, the model is aligned such that the PV system aligns with East and North directions, and the cloud direction $\alpha$ is defined relative to North. For PV systems with non-zero azimuth alignment, $\alpha$ in (6) should be replaced by:

$$
\alpha=\alpha_{N}-\alpha_{a z}
$$

where $\alpha_{N}$ is the measured cloud direction and $\alpha_{a z}$ is the azimuth angle of the solar power plant, both defined relative to North.

\section{Performance Evaluation}

The WCS-RR estimate is evaluated by the following performance metrics. First, we define the compliance indicator $\sigma$ by dividing the actual ramp rate by the corresponding WCS-RR estimate, as in:

$$
\sigma(t)=R R^{\text {actual }}(t) / R R^{\text {estimate }}(t) .
$$


When $\sigma \leq 1$, the actual ramp rate complies with the WCS-RR estimate. The maximum $\sigma$ in each non-overlapping evaluation window of length $m$ minutes is:

$$
\mu_{j}=\max \{\sigma(i) ; i \in[n j, n(j+1)-1]\}, j \in[1, N] .
$$

$n=(m \times 60) \mathrm{sec} / \mathrm{s}$ represents the number of $\sigma$ in the $j^{t h}$ evaluation window with a temporal resolution of $s \mathrm{sec}$, and $N$ is the total number of evaluation windows computed by rounding up the expression $T / m$, in which $T$ is the overall daily time window of WCS-RR in minutes. The selection of $m$ is somewhat arbitrary: a shorter window length results in more windows with exclusively clear or overcast conditions which are not of concern for ramp rates while longer window lengths tend to evaluate $\sigma$ too infrequently. Since transmission system operators are typically required to counteract power fluctuations with load following at a time scale of less than $30 \mathrm{~min}$, we apply window lengths of $m=2,10$, and $30 \mathrm{~min}$. The compliance rate $\phi$ is defined as:

$$
\phi=\frac{N_{c p l}}{N} \text {. }
$$

The number of compliance events $N_{c p l}$ indicates the number of windows that satisfy $\mu_{j} \leq 1$. Subsequently, the noncompliance rate becomes:

$$
\epsilon=(1-\phi) \times 100 \% \text {. }
$$

While risk-adverse actors would prefer that the WCS-RR always envelopes the observed ramps, excessive WCS-RR may result in an over-sized energy storage system. To quantify the extent to which the WCS-RR overpredicts the actual ramp, all compliance events are further evaluated by the degree of overestimation $\delta$ :

$$
\delta=\left(\frac{1}{N_{c p l}} \sum_{j \in N_{c p l}} 1-\mu_{j}\right) \times 100 \% .
$$

\section{VAlidation Setup AND Data InPUT}

\section{A. Experimental Setup and data}

To validate the proposed method, we set up an experiment at the University of California (UCSD) campus test bed. Figure 2 illustrates the layout of an existing PV systems located on the EBU2 building $\left(32^{\circ} 52^{\prime} 53.1^{\prime \prime} N\right.$, $117^{\circ} 13^{\prime} 59.2^{\prime \prime} \mathrm{W}$ ) at the campus of the UCSD with a tilt angle of $20^{\circ}$ and an azimuth angle of $225^{\circ}$ east of north. It is arranged in 5 arrays, consisting of a total of $210 \mathrm{PV}$ panels with the overall dimension of $38.2 \mathrm{~m} \times 15.2 \mathrm{~m}$ (marked by red in Figure 2). The total nominal power is $43 \mathrm{~kW} \mathrm{DC}$. The PV power was measured at a 2 sec sampling rate from September 29, 2017 to June 5, 2018 by 5 inverters (SMA Sunny Boy 7000US), with a total rated power of $35 \mathrm{~kW}$ AC. Excluding server shutdowns, rainy and overcast days, and clear days (defined as less than $30 \mathrm{~min}$ of cloud cover), 59 partially cloudy days remain, which are of interest to the experiment because partial cloud cover causes the largest power ramps. Note that the production field includes the ground area in between the rows, which mathematically enters (2) through the power plant dimensions. To avoid errors from clear sky and PV performance models, the power produced on the most recent clear day is used as the clear sky power.

Cloud speed and direction are required to relate cloud field to ramp rates. While the proposed model is compatible with any type of cloud speed measurement, we obtain cloud speed measurements from our in-house Cloud Speed Sensor (CSS), which provides an accurate yet affordable means to measure local cloud motion vectors (CMVs) [17], [18]. The instrument is installed on the same rooftop as the PV system and marked in Figure 2

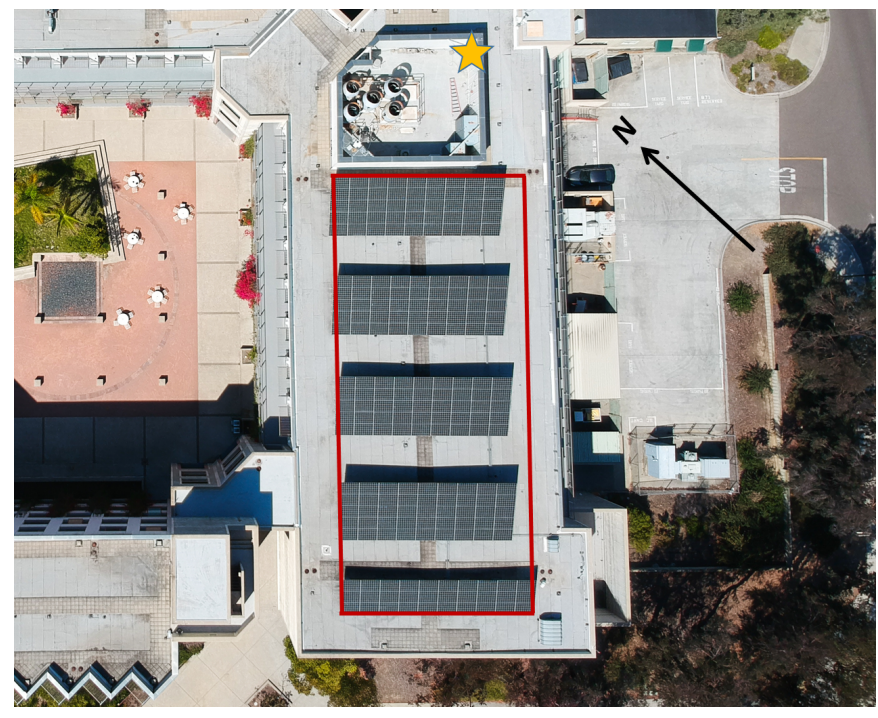

Fig. 2. Aerial view of the PV system installed on EBU2 at UCSD and the PV production field (red) considered in this paper. The yellow star indicates the CSS located 10 meters north of the upper corner of the PV system. CDrone Photo

\section{B. Search Time Window and Data Processing}

Since CMVs are only available in irregular intervals, a search window length needs to be defined to average the CMV data and select the $k t_{\text {min }}$. Longer windows challenge the assumption of cloud field homogeneity, causing older cloud fields that are likely different from the ones at present to be counted. Shorter windows may not contain sufficient cloud cover events and falsely suggest that clear conditions will persist. Based on our experience, the cloud field in coastal Southern California is typically steady over a few hours, so we consider a 30 min window centered at the time of interest as a conservative upper bound.

\section{RESUlTS AND Discussion}

\section{A. Performance on Two Sample Days}

In this section, two detailed examples are analyzed to illustrate and explain the performance of the proposed model. The WCS-RR estimate on Oct 8, 2017, one of the best days ( $100 \%$ compliance across all three evaluation windows), is first validated against the actual ramp rates in Figure 3. The 
top plot shows real PV power from 8:00 to 17:00 PDT and clear sky power output from 2 days earlier. The clouds are observed to move southward to eastward over the day with speeds ranging from 4 to $8 \mathrm{~m} \mathrm{~s}^{-1}$, as illustrated in the middle plot. The bottom plot illustrates the observed ramp rate and the WCS-RR estimates.

The WCS-RR generally is positively proportional to clear sky power and cloud speed, completely enveloping the actual ramp rate magnitude and timing. This day is characterized by frequent low scattered stratocumulus clouds, causing successive cloud optical thickness changes in a few time periods, subsequently the corresponding $k t$ variations influence the magnitude of the WCS-RR bound. There is a 1.5 hour exception from 12:00 to 13:30 PDT when the sky was completely clear, significantly lowering the WCS-RR estimate because the difference between 1 and $k t_{\text {min }}$ becomes minimal. Within $\pm 15 \mathrm{~min}$ of 13:00 PDT there is no WCSRR estimate because we do not have a valid cloud speed measurement under the clear sky cover to produce a WCSRR. The largest observed ramp rate of the day occurred at 13:46 PDT, caused by a sudden partial stratocumulus cloud cover after the prior 1.5 hour clear sky condition (Figure 4). Because this cloud condition change (clear to worst-case thick clouds) is the exact circumstance modeled in Equation (6), the WCS-RR estimate accurately captures the ramp magnitude with only a $5 \%$ overestimate.

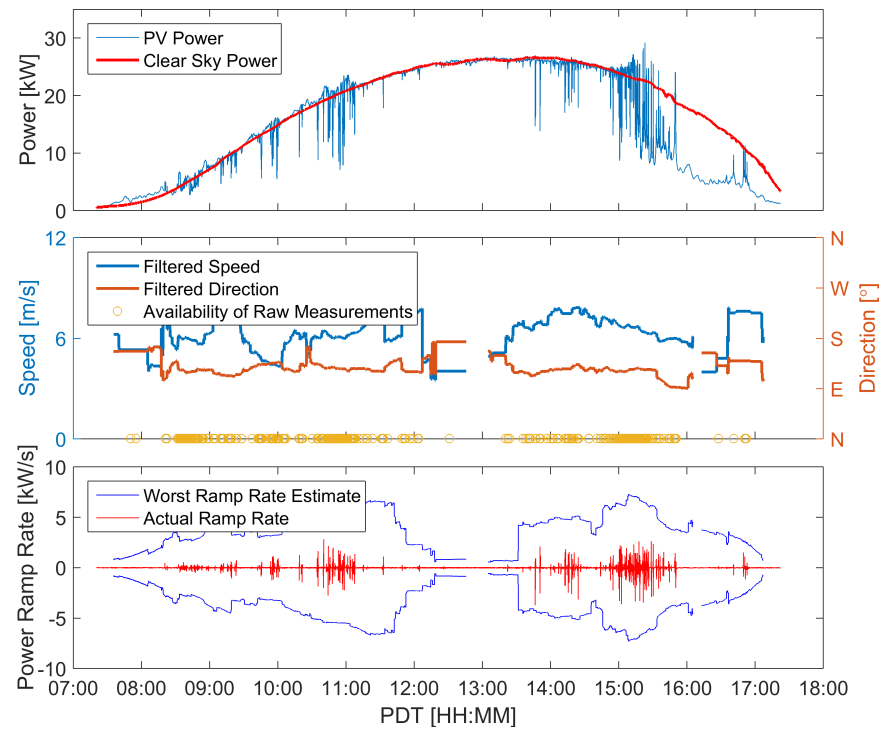

Fig. 3. Example validation of the proposed method on Oct 8, 2017. Top: Actual PV power on Oct 8, 2017 (blue) and on the most recent clear day (Oct 6, 2017, red). Middle: The velocity and directions derived from raw CSS CMV measurements (their availabilities are indicated by dots). Bottom: Comparison between actual ramp rate and WCS-RR estimate.

The daily noncompliance rate of $0 \%$ across all evaluation windows in this day confirms that the observed ramp rates are perfectly enveloped by the WCS-RR, at a cost of ramp rate overestimates from 56\% (30 min windows) to $70 \%$ (2 $\mathrm{min}$ windows). The ideal metrics would be $0 \%$ noncompliance and $0 \%$ overestimate. In reality, there is a trade-off between

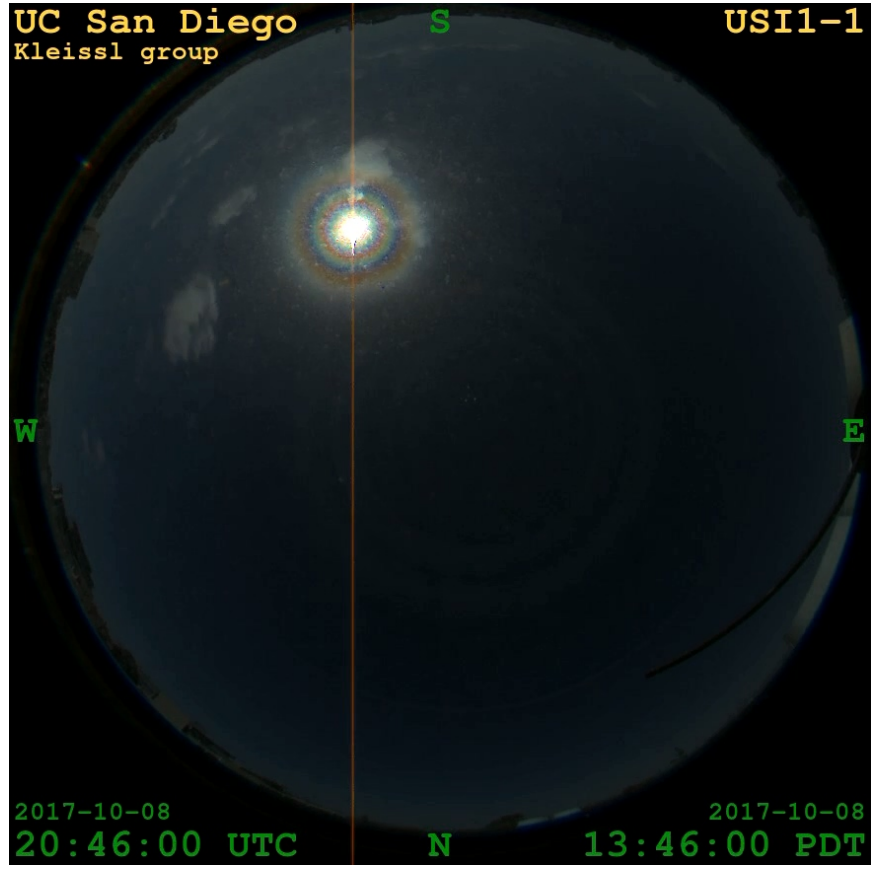

Fig. 4. Sky image of a cloud shading event at 13:46 PDT on Oct 8, 2017 with the largest ramp of the PV plant.

the noncompliance and the overestimate metrics: to bracket all large power ramps, the model conservatively assumes a cloud condition change from clear sky to thick clouds at all times, including periods with clear or overcast skies, which inevitably over-predicts the ramp rates. The only hypothetical scenario with $0 \%$ noncompliance and $0 \%$ overestimate would be for a series of thick clouds of the same size as the PV plant to pass the plant along $L$ (or analogously $W$ ); in those conditions, the plant would continually ramp up or down with a ramp rate equal to the WCS-RR. For Oct 8, 2017, the minimal overprediction of the largest ramp event (on 13:46 PDT) proves that the over-prediction is not excessive. The trade-off between the overestimate and noncompliance rate will be further discussed in the next section.

Also, May 9 is analyzed in Figure 5 as an example of a day with the noncompliance ramp events. On this day, there are 2 hour period in the late afternoon when the noncompliance ramp events occurred during high PV variability on 16:00 to 18:00 PDT, which can be explained by the cloud edge enhancement causing irradiances to be larger than the clearsky model (the top plot) due to additional diffuse irradiance [9]. While ramp rate violations are observed in those times, the actual ramp rate exceedances are relatively small (bottom plot). If the difference between the real PV output and the respective clear sky power were factored in, the bound of WCS-RR would be widening accordingly and sufficient to envelope the ramp rates. Nonetheless, the results still show that the true worst ramp rates generally follow the calculated WCS-RR estimates closely, and this example illustrates again that the accuracy of the proposed method primarily depends on the quality and availability of CMVs. 


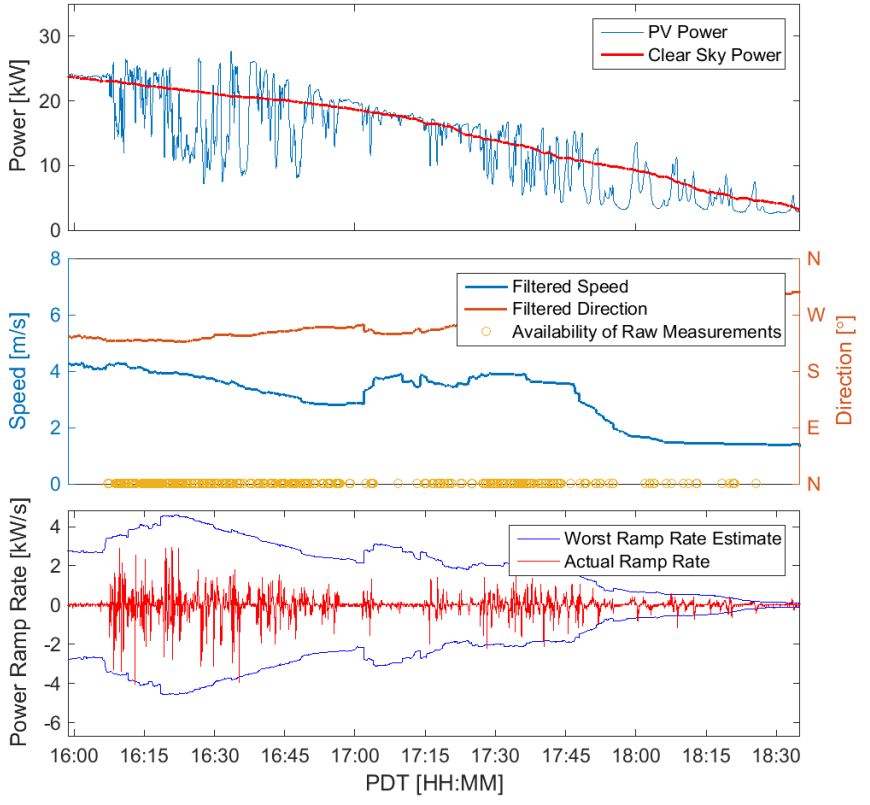

Fig. 5. Save layout as in Figure 3 but for May 9 illustrating noncompliance events caused by cloud edge enhancement effect.

\section{B. Aggregate Ramp Rate Statistics and Discussion}

The evaluation of the proposed method over an extensive set of 59 days is summarized in Table 1. Overall, the method shows promise: for the shortest 2 min window, the average noncompliance rate is only $3.1 \%$ at a cost of a $71.8 \%$ overestimate. The noncompliance rate slightly worsens with wider evaluation windows, which is expected as the chance that a noncompliance event $(\sigma>1)$ is included in the evaluation window increases with wider windows. Nevertheless, even under the longest $30 \mathrm{~min}$ evaluation window, the average noncompliance rate is $11.9 \%$. The degree of overestimation worsens with shorter evaluation window length (greater number of windows). Because WCSRR estimates are generally conservative at all times except the time of the daily largest ramp events, the degree of overestimate would be minimal when only a single time window (i.e. the daily largest ramp event) is considered over the day but tends to be larger when more evaluation windows are considered.

TABLE 1

Summary of Average Performance Metric over 59 Days

\begin{tabular}{|c|c|c|c|c|c|}
\hline $\begin{array}{c}\epsilon_{2 \min } \\
{[\%]}\end{array}$ & $\begin{array}{c}\delta_{2 \min } \\
{[\%]}\end{array}$ & $\begin{array}{c}\epsilon_{10 \min } \\
{[\%]}\end{array}$ & $\begin{array}{c}\delta_{10 \min } \\
{[\%]}\end{array}$ & $\begin{array}{c}\epsilon_{30 \min } \\
{[\%]}\end{array}$ & $\begin{array}{c}\delta_{30 \min } \\
{[\%]}\end{array}$ \\
\hline Average 3.1 & 71.8 & 7.6 & 63.4 & 11.9 & 56.4 \\
\hline
\end{tabular}

By examining the sky images for all noncompliance events, we conclude that the primary reasons for noncompliance events are few and/or inaccurate cloud speed measurements, which affects the performance of the WCS-RR method because CMV are key inputs to the WCS-RR (as per Equation (1)). However, these clouds may not be large enough to cause meaningful ramps. For large PV systems, we expect sparse or small clouds to be even less relevant as they cover only a fraction of the PV plant. We could achieve nearly flawless results if we limit the model more stringently to periods with robust CMV data, but this also inevitably limits WCS-RR output during some time periods.

\section{Limitations and Applications}

A physical limit for this method is that the sampling time needs to be such that the cloud velocity times the sampling time step does not exceed the dimension of the PV system in the direction of cloud motion. Geometrically, Equation (1) is only valid if $v \Delta t$ is less than $W /|\cos \alpha|$ and $L /|\sin \alpha|$. For any given sampling rate and dimension of the PV system, there is a cloud velocity for which that sampling rate starts to become too slow, and the WCS-RR then incorrectly predicts the ramp rate over the sampling time step. Since cloud velocity has a physical limit, a conservative sampling rate can be derived given the dimensions of the PV system. Large PV systems can tolerate larger sampling rates.

The WCS-RR estimate is also briefly influenced by the additional diffuse irradiance resulted from the cloud edge enhancement, as illustrated in the earlier section. To countereffect the irradiance deviation, the modeled clear sky power may need to be upscaled by a small factor.

The tradeoff between noncompliance rate and degree of overestimation deserves further discussion. For example, an energy storage system that can mitigate larger ramp rates is more costly, but a smaller energy storage system may not mitigate all ramps and result in noncompliance penalties and/or curtailed solar energy. Ultimately, economic modeling specific to a project is needed to answer these questions. In such conditions, a safety factor could be introduced in Equation (6) to determine appropriate $k t_{\min }$ and accordingly adjust WCS-RR to fit the risk profile of the investor.

\section{CONCLUSION}

Knowing the maximum expected photovoltaic (PV) production ramp rate proves useful for the design of $\mathrm{PV}$ and energy storage system (ESS) by determining the ESS energy reserve required to offset power fluctuations. The main goal of this paper is to provide a method to inform optimal design of a solar power plant with ESS by estimating the worst-case scenario ramp rates (WCS-RR) in the design phase, prior to the PV installation and in the absence of local high-frequency irradiance data. A method to estimate WCS-RR for a PV plant, based on cloud speed and direction, solar irradiance (or power), and geometric PV plant layout is developed and validated. The principal assumption is that the cloud field properties are stationary during the cloud passage over the plant. WCS-RR is validated against a PV system during a 8month period, using cloud motion vectors (CMVs) measured by our in-house cloud speed sensor. The actual ramp rates comply with the calculated maximum ramp rates $96.9 \%$ of the time with 2 min evaluation time window. The remaining $3.1 \%$ of times can be primarily ascribed to inaccurate cloud velocity measurements in conditions with sparse and/or thin 
clouds and cloud edge enhancement effects. In conclusion, the method proposed in this study will be helpful for both PV plant planning and operation.

\section{ACKNOWLEDGEMENT}

Juan Luis Bosch has been financed in part by Projects ENE2017-83790-C3-3-R and ENE2014-59454-C3-2-R which were funded by the Ministerio de Ciencia, Innovacin $\mathrm{y}$ Universidades and Ministerio de Economa y Competitividad, respectively, and co-financed by the European Regional Development Fund. In addition, Íñigo de la Parra has been partially supported by the Spanish State Research Agency (AEI) and FEDER-UE under grants DPI2016-80641-R and DPI2016-80642-R.

\section{REFERENCES}

[1] V. Gevorgian and S. Booth, "Review of prepa technical requirements for interconnecting wind and solar generation," tech. rep., National Renewable Energy Lab.(NREL), Golden, CO (United States), 2013.

[2] Y. Ru, J. Kleissl, and S. Martinez, "Exact sizing of battery capacity for photovoltaic systems," European Journal of Control, vol. 20, no. 1, pp. 24-37, 2014.

[3] J. Marcos Álvarez, Í. d. 1. Parra Laita, M. García Solano, and L. Marroyo Palomo, "Control strategies to smooth short-term power fluctuations in large photovoltaic plants using battery storage systems," Energies, 2014, 7 (10), 6593-6619, 2014.

[4] R. Tonkoski, L. A. Lopes, and T. H. El-Fouly, "Coordinated active power curtailment of grid connected pv inverters for overvoltage prevention," IEEE Transactions on sustainable energy, vol. 2, no. 2, pp. 139-147, 2010.

[5] S. Shivashankar, S. Mekhilef, H. Mokhlis, and M. Karimi, "Mitigating methods of power fluctuation of photovoltaic (pv) sources-a review," Renewable and Sustainable Energy Reviews, vol. 59, pp. 1170-1184, 2016.

[6] A. Nguyen, M. Velay, J. Schoene, V. Zheglov, B. Kurtz, K. Murray, B. Torre, and J. Kleissl, "High pv penetration impacts on five local distribution networks using high resolution solar resource assessment with sky imager and quasi-steady state distribution system simulations," Solar Energy, vol. 132, pp. 221-235, 2016.

[7] M. Saleh, L. Meek, M. A. Masoum, and M. Abshar, "Battery-less shortterm smoothing of photovoltaic generation using sky camera," IEEE Transactions on Industrial Informatics, vol. 14, no. 2, pp. 403-414, 2017.

[8] R. Perez, S. Kivalov, J. Schlemmer, K. Hemker Jr, D. Renné, and T. E. Hoff, "Validation of short and medium term operational solar radiation forecasts in the us," Solar Energy, vol. 84, no. 12, pp. 2161-2172, 2010.

[9] M. Lave, J. Kleissl, and E. Arias-Castro, "High-frequency irradiance fluctuations and geographic smoothing," Solar Energy, vol. 86, no. 8, pp. 2190-2199, 2012.

[10] M. Lave, M. J. Reno, and R. J. Broderick, "Characterizing local highfrequency solar variability and its impact to distribution studies," Solar Energy, vol. 118, pp. 327-337, 2015.

[11] R. Perez, M. David, T. E. Hoff, M. Jamaly, S. Kivalov, J. Kleissl, P. Lauret, M. Perez, et al., "Spatial and temporal variability of solar energy," Foundations and Trends® in Renewable Energy, vol. 1, no. 1, pp. 1-44, 2016.

[12] J. Marcos, L. Marroyo, E. Lorenzo, D. Alvira, and E. Izco, "From irradiance to output power fluctuations: the pv plant as a low pass filter," Progress in Photovoltaics: Research and Applications, vol. 19, no. 5, pp. 505-510, 2011.

[13] M. Lave, J. Kleissl, and J. S. Stein, "A wavelet-based variability model (wvm) for solar pv power plants," IEEE Transactions on Sustainable Energy, vol. 4, no. 2, pp. 501-509, 2012.

[14] M. Lave and J. Kleissl, "Cloud speed impact on solar variability scaling-application to the wavelet variability model," Solar Energy, vol. 91, pp. 11-21, 2013.

[15] J. Marcos, Í. de la Parra, M. García, and L. Marroyo, "Simulating the variability of dispersed large pv plants," Progress in Photovoltaics: Research and Applications, vol. 24, no. 5, pp. 680-691, 2016.
[16] P. Ineichen and R. Perez, "A new airmass independent formulation for the linke turbidity coefficient," Solar Energy, vol. 73, no. 3, pp. 151157, 2002.

[17] V. Fung, J. Bosch, S. Roberts, and J. Kleissl, "Cloud shadow speed sensor," Atmospheric Measurement Techniques, vol. 7, no. 6, pp. 1693$1700,2014$.

[18] G. Wang, B. Kurtz, and J. Kleissl, "Cloud base height from sky imager and cloud speed sensor," Solar Energy, vol. 131, pp. 208-221, 2016. 